\title{
Is cardiac magnetic resonance ready for aortic regurgitation?
}

\author{
Michael Markl1,2, Jeesoo Lee', Maurice Pradella ${ }^{1,3}$
}

'Department of Radiology, Northwestern University, Feinberg School of Medicine, Chicago, Illinois, United States of America 2Department of Biomedical Engineering, Northwestern University, McCormick School of Engineering, Evanston, Illinois, United States of America ${ }^{3}$ Department of Radiology, University Hospital Basel, University of Basel, Basel, Switzerland

\section{Related article}

by Haberka et al., see p. 965
Current European Society of Cardiology and American Heart Association guidelines highlight the importance of echocardiography for the assessment of aortic regurgitation (AR), since it is widely available and allows for comprehensive evaluation of multiple factors in one exam, such as valve morphology, jet angle, pressure half time as well as quantification of regurgitant volume (RVol) and regurgitant fraction (RF), left ventricular (LV) dimensions and function [1, 2]. However, standard 2D echocardiography is often limited by anatomic coverage, and limited inter-, intra-observer, and sonographer variability. As a diagnostic alternative, cardiac magnetic resonance imaging (CMR) offers superior image contrast with flexible $2 \mathrm{D}$ or $3 \mathrm{D}$ image orientation and is considered the clinical reference standard for LV and right ventricular (RV) volumetry [3]. In addition, 2D phase-contrast CMR can reliably measure blood flow in the aorta and has been shown to provide reproducible AR RVol and $\mathrm{RF}$, which are crucial metrics associated with the heart valve disease severity [4]. However, despite the benefits of CMR over transthoracic echocardiography (TTE), only limited data on the systematic comparison of both modalities have been presented.

In the current issue of Kardiologia Polska (Polish Heart Journal), Haberka et al. present an interesting and timely study comparing TTE and CMR for the assessment of AR in a group of 49 patients [5]. The study cohort included a broad range of AR severity determined by the quantitative and semiquantitative integrative approach described in American Society of Echocardiography guidelines [3]. AR severity for all patients was also assessed using RVol and RF quantified by 2D phase-contrast CMR with the same cutoffs for the grading used by TTE. In line with previous studies $[4,6,7]$, the authors demonstrated that TTE overestimated AR severity, RVol, and RF in comparison to CMR. Further investigation revealed that factors associated with AR grading discrepancies between CMR and TTE were the presence of eccentric AR flow jets ( $\geq 40^{\circ}$ deviation from the axis perpendicular to the aortic valve). This finding illustrates a well-known limitation of 2D Doppler echocardiography which can only quantify the AR flow jet velocity component parallel to the ultrasound beam. As a result, the increased eccentricity of the AR flow jets can compromise the accuracy of TTE. However, whether the presence of an eccentric jet leads to over- or underestimation by TTE was not discussed by the authors. In addition to AR assessment, the authors compared the diagnostic value of CMR vs TTE for detecting LV remodeling impacted by AR. Their study found that, for both modalities, LV end-diastolic volume (EDV) significantly correlated with RVol, which supports the potential benefit of using EDV as an indicator of LV dilatation associated with AR $[8,9]$. Furthermore, LV EDV and ejection fraction (LVEF) were higher for CMR compared to TTE. Since LV EDV and LVEF are both used to determine (surgical) intervention, this finding implies that CMR might be preferable in monitoring the progression of AR.

There are a few limitations: As the authors mentioned, there were only 5 cases of severe $A R$, which limits the clinical transferability of results. Second, for this type of study, it is of importance to ensure the discrepancy of measurements is primarily driven by the difference 
in the technique itself and not by physiological conditions between TTE and CMR examination. Blood pressure, heart rate, and time between the TTE and CMR exams would have been good indicators for changes in subject physiology but were not reported. Third, accurate flow quantification use of $2 \mathrm{D}$ phase-contrast $C M R$ requires careful $2 \mathrm{D}$ analysis plane orientation [10] along the aorta [11], correction for eddy-current induced background phase offset [12], and the use of sufficiently high-velocity encoding sensitivity. Finally, only half of the study cohort $(n=24)$ were used to compare AR flow metrics between the two modalities due to the limited applicability of the proximal isovelocity surface area (PISA) method. PISA is less suitable for AR than mitral regurgitation as the visualization of flow convergence in color Doppler echocardiography is often restricted by thickening and/or calcification of the aortic valve which is also the case in this study cohort. As the focus was to compare TTE vs CMR, other indirect volumetric methods (e.g., subtracting mitral valve stroke volume from LV stroke volume) [3] available by TTE could have been an alternative to PISA in order to improve the statistical power of the study results.

Nonetheless, the data presented in this study by Haberka et al. adds valuable information to understand the differences between the two modalities in AR assessment. Further investigation is still required to demonstrate the prognostic value of CMR-based AR assessment. TTE will thus likely remain the first-choice imaging modality to evaluate and follow-up patients with AR in clinical practice due to its wide availability. Nevertheless, the advantages of CMR are evident in its ability to provide robust and reproducible aortic flow quantification and assessment of global LV and RV function metrics. CMR could thus serve as an important clinical tool for patients with severe AR who require an accurate and comprehensive diagnosis to determine the need and timing of intervention.

\section{Article information}

Acknowledgements: Maurice Pradella is supported by the Bangerter-Rhyner Foundation and Freiwillige Akademische Gesellschaft Basel. Conflict of interest: None declared.

Open access: This article is available in open access under Creative Common Attribution-Non-Commercial-No Derivatives 4.0 International (CC BY-NC-ND 4.0) license, allowing to download articles and share them with others as long as they credit the authors and the publisher, but without permission to change them in any way or use them commercially. For commercial use, please contact the journal office at kardiologiapolska@ptkardio.pl.

How to cite: MarkI M, Lee J, Pradella M. Is cardiac magnetic resonance ready for aortic regurgitation?. Kardiol Pol. 2021; 79(9): 945-946, doi: 10.33963/KP.a2021.0094.

\section{REFERENCES}

1. Baumgartner H, Falk V, Bax JJ, et al. 2017 ESC/EACTS Guidelines for the management of valvular heart disease. Eur Heart J. 2017; 38(36): 2739 2791, doi: 10.1093/eurheartj/ehx391, indexed in Pubmed: 28886619.

2. Otto CM, Nishimura RA, Bonow RO, et al. 2020 ACC/AHA Guideline for the Management of Patients With Valvular Heart Disease: A Report of the American College of Cardiology/American Heart Association Joint Committee on Clinical Practice Guidelines. Circulation. 2021; 143(5): e72-e7e227, doi: 10.1161/CIR.0000000000000923, indexed in Pubmed: 33332150.

3. Zoghbi W, Adams D, Bonow R, et al. Recommendations for noninvasive evaluation of native valvular regurgitation $A$ report from the american society of echocardiography developed in collaboration with the society for cardiovascular magnetic resonance. J Indian Acad Echocardiogr Cardiovasc Imaging. 2020; 4(1): 58, doi: 10.4103/2543-1463.282191.

4. Cawley PJ, Hamilton-Craig C, Owens DS, et al. Prospective comparison of valve regurgitation quantitation by cardiac magnetic resonance imaging and transthoracic echocardiography. Circ Cardiovasc Imaging. 2013; 6(1): 48-57, doi: 10.1161/CIRCIMAGING.112.975623, indexed in Pubmed: 23212272.

5. Haberka M, Bałys M, Gąsior Z, et al. Aortic regurgitation and left ventricle remodeling on cardiac magnetic resonance and transthoracic echocardiography. Kardiol Pol. 2021; 79(9): 965-971, doi: 10.33963/KP.a2021.0047, indexed in Pubmed: 34176113.

6. Gelfand EV, Hughes $\mathrm{S}$, Hauser $\mathrm{TH}$, et al. Severity of mitral and aortic regurgitation as assessed by cardiovascular magnetic resonance: optimizing correlation with Doppler echocardiography. J Cardiovasc Magn Reson. 2006; 8(3): 503-507, doi: 10.1080/10976640600604856, indexed in Pubmed: 16755839.

7. Kammerlander AA, Wiesinger M, Duca F, et al. Diagnostic and prognostic utility of cardiac magnetic resonance imaging in Aortic regurgitation. JACC Cardiovasc Imaging. 2019; 12(8 Pt 1): 1474-1483, doi: 10.1016/j. jcmg.2018.08.036, indexed in Pubmed: 30448117.

8. Myerson SG, d'Arcy J, Mohiaddin R, et al. Aortic regurgitation quantification using cardiovascular magnetic resonance: association with clinical outcome. Circulation. 2012; 126(12): 1452-1460, doi: 10.1161/CIRCULATIONAHA.111.083600, indexed in Pubmed: 22879371.

9. Uretsky S, Argulian E, Supariwala A, et al. Quantification of left ventricular remodeling in response to isolated aortic or mitral regurgitation. J Cardiovasc Magn Reson. 2010; 12(11): 32-1088, doi: 10.1186/1532-429X-12-32, indexed in Pubmed: 20497540.

10. Tang C, Blatter DD, Parker DL. Accuracy of phase-contrast flow measurements in the presence of partial-volume effects. J Magn Reson Imaging. 1993; 3(2): 377-385, doi: 10.1002/jmri.1880030213, indexed in Pubmed: 8448400 .

11. Iwamoto $Y$, Inage $A$, Tomlinson $G$, et al. Direct measurement of aortic regurgitation with phase-contrast magnetic resonance is inaccurate: proposal of an alternative method of quantification. Pediatr Radiol. 2014; 44(11): 1358-1369, doi: 10.1007/s00247-014-3017-x, indexed in Pubmed: 24939669.

12. Gatehouse PD, Rolf MP, Graves MJ, et al. Flow measurement by cardiovascular magnetic resonance: a multi-centre multi-vendor study of background phase offset errors that can compromise the accuracy of derived regurgitant or shunt flow measurements. J Cardiovasc Magn Reson. 2010; 12: 5, doi: 10.1186/1532-429X-12-5, indexed in Pubmed: 20074359. 